\title{
A partial consequence account of truthlikeness *
}

\author{
Gustavo Cevolani† and Roberto Festa ${ }^{\ddagger}$
}

8 September 2018

\begin{abstract}
Popper's original definition of truthlikeness relied on a central insight: that truthlikeness combines truth and information, in the sense that a proposition is closer to the truth the more true consequences and the less false consequences it entails. As intuitively compelling as this definition may be, it is untenable, as proved long ago; still, one can arguably rely on Popper's intuition to provide an adequate account of truthlikeness. To this aim, we mobilize some classical work on partial entailment in defining a new measure of truthlikeness which satisfies a number of desiderata. The resulting account has some interesting and surprising connections with other accounts on the market, thus shedding new light on current attempts of systematizing different approaches to verisimilitude.
\end{abstract}

Keywords: truthlikeness, verisimilitude, information, content, consequence, similarity, Schurz and Weingartner, Oddie.

\section{Contents}

1 Introduction 2

2 Defining truthlikeness: three approaches 3

3 Truthlikeness as (partial) information about the truth $\quad 6$

3.1 The basic feature approach to truthlikeness . . . . . . 8

3.2 From "categorical" to "partial" information . . . . . . . . . 9

4 Discussion and comparison with other accounts 12

4.1 A surprising equivalence . . . . . . . . . . . . . 13

4.2 Truthlikeness and logical strength . . . . . . . . . . . 15

${ }^{*}$ Final preprint draft. Published as: G. Cevolani and R. Festa (2018). "A partial consequence account of truthlikeness". In: Synthese. URL: 10.1007/s11229-018-019473.

${ }^{\dagger}$ IMT School for Advanced Studies Lucca, Piazza San Francesco 19, 55100 Lucca (Italy). E-mail: gustavo.cevolani@imtlucca.it

${ }^{\ddagger}$ Department of Humanistic Studies University of Trieste, Via Principe di Montfort 3, 34124 Trieste (Italy). E-mail: festa@units.it 


\section{Introduction}

Intuitively, a proposition (or theory, or hypothesis) $h$ is "close to the truth" when it tells many things about the relevant domain, and many of these things are true, or at least approximately true. In this sense, truthlikeness or verisimilitude is "a mixture of truth and information", as Oddie (1986, p. 12) puts it: the more true information $h$ provides about the domain, the closer to "the whole truth" $h$ is (cf. Popper 1963, p. 237).

Starting in the seventies of the past century, logicians and philosophers of science proposed a number of competing formal explications of the above intuition (for surveys, see Niiniluoto 1998 and Oddie 2016). As a result of this ongoing work, quite different accounts of truthlikeness are currently on offer. More recent research has increasingly focused on the attempt of classifying and comparing such accounts in an abstract, general way (see, e.g., Zwart 2001, Niiniluoto 2003, Zwart and Franssen 2007, Schurz and Weingartner 2010, and Oddie 2013). This led to both refining and revising the informal distinction between "content" and "likeness" approaches that was routinely employed to classify different accounts of truthlikeness (cf., e.g., Oddie 1986, p. x). In particular, Gerhard Schurz has convincingly argued for the importance of recognizing a third, "consequence" approach to defining verisimilitude, based on a "conjunctive" representation of propositions as sets of (relevant) consequences, in contrast with a "disjunctive" view in terms of possible worlds or state descriptions (Schurz and Weingartner 2010; Oddie 2013).

In this paper, we introduce and discuss a novel truthlikeness measure with the following features: it fits nicely within a conjunctive and consequencebased approach to verisimilitude as the one advocated by Schurz; it satisfies most adequacy conditions discussed in the literature; and finally, as it turns out somehow surprisingly, it is equivalent to a well known measure proposed within one standard disjunctive and likeness-based approach to truthlikeness (the so called Tichý-Oddie's average measure). Our results thus shed new light on current work on analyzing and systematizing different and competing approaches to verisimilitude.

We proceed as follows. Section 2 presents the fundamental ideas concerning truthlikeness and the classification of different approaches. In section 3 we introduce a novel truthlikeness measure and discuss its main properties. The main result of the paper comes in section 4, where the equivalence between our measure and Tichý-Oddie's "average" measure is proved. In this section, we also discuss the relationship between our account and other ap- 
proaches, including Schurz and Weingartner's "relevant element" definition of truthlikeness and Niiniluoto's similarity-based account. Finally, section 5 contains some concluding remarks and directions for future work. The proofs of all main claims in the paper appear in the final appendix.

\section{Defining truthlikeness: three approaches}

Two friends, Henry and Gloria, are planning a trip and wonder about the weather they are going to find at their destination. Henry believes that it will be hot, rainy, and windy (call $h$ this conjunction); Gloria is more optimistic, and believes $g$, that it will be hot, dry, and windy. In short, they agree they shall have a hot and windy day, but disagree about whether it will rain or not. Now suppose that, as a matter of fact, on their arrival the weather is actually cold, rainy and windy: this represents "the whole truth", call it $t$, about the weather conditions in the relevant place and time. It is now clear that Gloria made a mistake in predicting no rain, and that Henry was right. On the other hand, they were both wrong in believing that it would be hot. Still, it seems clear that Henry was closer to the truth than Gloria was: he made only one mistake ("hot" instead of "cold"), while she erred on two counts ("hot" and "dry"). In other words, it seems natural to say that while both $h$ and $g$ are false propositions, $h$ is a better approximation to the truth $t$ than $g$ is.

The first goal of a theory of truthlikeness is to make sense of assessments of relative closeness to the truth like the one considered above. All current accounts of verisimilitude deliver such assessments for at least some of the propositions of the relevant languages. Still, they disagree quite wildly on how truthlikeness should be defined and evaluated. To illustrate, consider the following small sample of basic principles, proposed by different participants to the debate as adequacy conditions for a definition of truthlikeness (for discussion, see especially Niiniluoto 1987, $232 \mathrm{ff}$. and Oddie 2013): given two distinct propositions $h$ and $g$,

P1 If $h$ and $g$ are false, $h$ may be closer to the truth than $g$;

P2 If $h$ is false and $g$ is true, $h$ may be closer to the truth than $g$;

P3 If $h$ and $g$ are true, and $h$ entails $g, h$ is closer to the truth than $g$;

P4 If $h$ and $g$ are false, and $g$ entails $h, h$ may be closer to the truth than $g$.

$\mathrm{P} 1$ requires that false propositions can differ in truthlikeness, as in the simple example given before. P2 says that sometimes a falsehood may be better (i.e., more verisimilar) than a truth: for instance, if the weather is actually cold, rainy and windy, saying (falsely) that it is hot, rainy and windy 
may be better than simply saying it is either cold or rainy, which is true but not very informative. Indeed, truthlikeness is a "game of excluding falsity and preserving truth", as Niiniluoto (1999, p. 73) puts it, and depends on the relative amount of true and false information provided about the world. Condition P3 requires that truthlikeness increases with logical strength among true propositions, while $\mathrm{P} 4$ denies this must be the case for false propositions.

Interestingly, with the only exception of $\mathrm{P} 1$ all other requirements are violated by at least one of the approaches to truthlikeness currently on the market (see Cevolani 2017 for discussion). In early work on truthlikeness, it has been customary to classify such approaches as either "content-based" or "likeness-based" (see, e.g., Oddie 1986, p. x). Roughly, according to the former the truthlikeness of $h$ only depends on the informative content and the truth-value of $h$; according to the latter, truthlikeness is a matter of how similar a proposition actually is to the complete description of the truth about the relevant domain. A couple of examples will serve to illustrate the essential intuitions behind each of them.

Within a content approach, the relative verisimilitude of two propositions $h$ and $g$ can be easily assessed at least in two "easy" cases (in most of the other cases, propositions can well be incomparable in truthlikeness). First, suppose that $h$ and $g$ are equally informative, but $h$ is true and $g$ false: then $h$ is more verisimilar than $g$. For instance, in our weather example, "rainy" is as informative as its negation "dry", but the former is true and hence more verisimilar than the latter, which is false. Second, suppose that $h$ and $g$ have the same truth value, but $h$ is more informative than $g$ : then, $h$ is also more truthlike than $g$. For instance, both "cold and rainy" and "cold" are true propositions, but the former is more verisimilar than the latter. This immediately implies that content accounts will meet condition P3: if $h$ and $g$ are both true, and $h$ entails $g$ (and, in this sense, $h$ is more informative), $h$ is more verisimilar than $g$. By the same reasoning, however, if $h$ and $g$ are both false, and $h$ entails $g, h$ is again more verisimilar than $g$, thus violating condition $\mathrm{P} 4$. This latter result is known in the literature as the child's play objection (cf. Tichý 1974, 157, fn. 2): if P4 fails, the truthlikeness of a false proposition can be increased just by strengthening it with some trivial falsehood.

Turning now to likeness approaches, they start by defining a distance measure (or at least a comparative notion of relative closeness) among the possible states of affairs characterizing the relevant domain (i.e., the possible worlds or their linguistic descriptions, see below). Then, the truthlikeness of $h$ is a function of the distances between the actual state of affairs and those possible worlds which are compatible with $h$. If this function is sufficiently sophisticated, the resulting explication will vindicate intuitive assessments of truthlikeness and eschew the child's play objection, by satisfying condition P4 (we shall see an example in section 4). Still, it is possible that P3 is now 
violated: for instance, according to Oddie (2013)'s characterization, likenessbased accounts meet P4 but violate P3 (however, other likeness approaches, like the one proposed by Niiniluoto 1987, meet both P3 and P4).

This informal characterization of content and likeness approaches is already sufficient to emphasize important differences between them. One, as Zwart (2001) first noted, concerns the following question: given that the truth $t$ itself is of course the most verisimilar proposition, which one has the least degree of truthlikeness? The two approaches give different answers. Since $\neg t$, the negation of the truth, is the weakest, and hence least informative, false proposition, it is also the least truthlike for content approaches. Within a likeness approach, instead, this role is reserved to the "complete falsehood" $f$, i.e., to the proposition which is the farthest from the truth $t$-like "hot, dry, and still" in our initial example.

The attempt to use the distinction above to classify different accounts within the content and likeness camps (Zwart 2001; Zwart and Franssen 2007) has been criticized by both Schurz and Weingartner (2010) and Oddie (2013). In particular, Schurz, followed on this by Oddie, has convincingly argued for the need of recognizing a third kind of approach to defining truthlikeness, different from both content and likeness approaches. Within this "consequence" approach, the truthlikeness of $h$ depends on the balance of the true and false propositions entailed by $h$ or, in other words, on the relative amount of true and false information conveyed by $h$ about the world. It follows that a consequence approach will agree with a contentbased one in accepting P3, i.e., the idea that, among truths, verisimilitude increases with informativeness, since a more informative proposition will have in this case more true consequences. On the other hand, it will agree on $\mathrm{P} 4$ with likeness-based accounts, since having more false consequences can well translate into lower overall truthlikeness. In sum, according to Schurz, consequence-based accounts combine the most appealing intuitions underlying the two alternative, content and the likeness, approaches. As we shall see in a moment, Popper's original account, even if flawed, falls squarely among the consequence-based accounts (Schurz and Weingartner 2010; Oddie 2013). Another example is Schurz's own "relevant element" account of truthlikeness, which represents a refined improvement of Popper's definition, avoids its defects, and will be discussed in section 4 .

In this connection, Schurz has proposed a further interesting distinction, that between conjunctive and disjunctive approaches to theory representation (Schurz and Weingartner 2010; Schurz 2011). The key idea is that the way in which propositions, or theories, are represented in the first place can have significant implications on how their relative verisimilitude is defined and assessed. Within a conjunctive approach, a proposition $h$ is represented as a conjunction of minimal "content parts", i.e., of the smallest items of information provided by $h$ on the world; as an example, $h$ may be the conjunction of its consequences in some language. A disjunctive approach 
instead represents $h$ as a disjunction of maximal "alternative possibilities", like the possible worlds or the models of the underlying language. ${ }^{1}$ According to Schurz, conjunctive approaches to theory representation are intrinsically more plausible and cognitively more manageable than disjunctive ones. Schurz's own approach, as well as consequence-based accounts in general, assume a conjunctive view of propositions and theories.

It is worth noting that Schurz's conjunctive/disjunctive distinction is somehow orthogonal to the content/likeness distinction already discussed above. For instance, both Oddie's and Niiniluoto's likeness-based account employ a disjunctive approach to theory representation. However, also Miller's and Kuipers' content approaches are disjunctive in this sense (Miller 1978; Kuipers 2000). On the other hand, Cevolani (2016) has shown how a conjunctive view can lead to a content-based ordering of truthlikeness. We shall come back to these issues in the final section 5 . In the next section, we present a consequence-based account of truthlikeness, based on a conjunctive representation of propositions, which however turns out to have surprising relationships with the disjunctive, likeness-based approaches.

\section{Truthlikeness as (partial) information about the truth}

Popper's definition of verisimilitude was based on apparently a sound intuition: the more true consequences and the less false consequences a theory or hypothesis $h$ has, the greater its verisimilitude. More precisely, let $C n(h)$ denote the class of logical consequences of $h$. Moreover let $C n_{T}(h)$ denote the class of its true consequences, and $C n_{F}(h)$ the class of its false consequences, to the effect that $C n_{T}(h) \cup C n_{F}(h)=C n(h)$. Then, according to Popper (1963, p. 233), $h$ is at least as close to the truth as $g$ iff $h$ has no less true consequences (and possibly more) and no more false consequences (and possibly less) than $g$ :

$$
C n_{T}(h) \supseteq C n_{T}(g) \text { and } C n_{F}(h) \subseteq C n_{F}(g)
$$

Moreover, $h$ is closer to the truth than $g$ if at least one of the two above inclusion relations is strict. It is well-known that Popper's definition is untenable, due to the so-called Tichý-Miller theorem. In fact, Miller (1974) and Tichý (1974) independently proved that no false theory $h$ can be closer to the truth than another (true or false) theory $g$ according to Popper's

\footnotetext{
${ }^{1}$ Of course, this distinction parallels the familiar one between two equivalent ways of expressing sentences in formal languages, namely, the one between conjunctive and disjunctive normal forms. As immaterial as this distinction may be from a purely logical point of view, it can have significant implications for the formal analysis of epistemological concepts, as already Carnap (1950, secs. 72-73, especially p. 407) observed.
} 
definition; i.e., that both $\mathrm{P} 1$ and $\mathrm{P} 2$ are violated. This proved fatal for Popper's explication of verisimilitude, showing it worthless for the very purpose Popper devised it: ordering false theories according to their closeness to the truth.

All accounts proposed within the post-Popperian research program on truthlikeness remedy the above defect with Popper's original definition. Some of them also retain the fundamental Popperian intuition: that the truthlikeness of $h$ balance the amount of true information and of false information entailed by $h$. One example is our own "basic feature" approach to truthlikeness, initially presented in Cevolani, Crupi, and Festa (2011) (see also Cevolani, Festa, and Kuipers 2013). Before presenting it, we introduce a small amount of terminology and notation.

We shall frame all relevant definitions in terms of a finite propositional language $\mathcal{L}_{n}$ with $n$ atomic propositions (cf. e.g. Zwart 2001; Niiniluoto 2003; Schurz and Weingartner 2010). Within $\mathcal{L}_{n}$, one can express $2^{2^{n}}$ logically non-equivalent propositions, including the tautological and the contradictory ones, denoted $T$ and $\perp$ respectively. Given two propositions $h$ and $g, h$ is said to be logically stronger than $g$ when $h$ entails $g$ but $g$ doesn't entail $h$ (in symbols: $h \vDash g$ but $g \not \models h$ ); thus, $\perp$ is the logically strongest proposition, and $T$ the weakest one.

Among the contingent (i.e., neither tautological nor contradictory), or factual, propositions of $\mathcal{L}_{n}$, some play a special role and deserve special mention. A basic proposition is an atomic proposition or its negation (a so called literal). A consistent conjunction $h$ of $m$ basic propositions ( $0 \leq$ $m \leq n$ ) will be called a conjunctive proposition of $\mathcal{L}_{n}$. If $m=0$, then $h$ is tautological; if $m=1$, it is a basic statement; and if $m=n$, it is a so called constituent (or state description) of $\mathcal{L}_{n}$. There are $2^{n}$ constituents, which are the logically strongest factual propositions of $\mathcal{L}_{n}$. The set $R(h)$ of constituents entailing $h$ (or, equivalently, the class of possible worlds in which $h$ is true) is called the range of $h$.

By definition, each constituent is logically incompatible with any other, and only one of them is true; this is denoted $t$ and is the strongest true statement expressible in $\mathcal{L}_{n}$. Intuitively, a constituent completely describes a possible state of affairs of the relevant domain (a "possible world"); thus, $t$ can be construed as "the (whole) truth" in $\mathcal{L}_{n}$, i.e., as the complete true description of the actual world. When one of the constituents of $\mathcal{L}_{n}$ is identified with the truth $t$, it partitions the set of propositions of $\mathcal{L}_{n}$ into the class $T=C n(t)$ of the true ones and its complement $F$ containing the false ones. Among these, the "complete falsehood" is represented by the "worst" constituent $f$ of $\mathcal{L}_{n}$, which is the conjunction of the negations of all true basic propositions, i.e., of all the conjuncts of $t$. 


\subsection{The basic feature approach to truthlikeness}

Within the basic feature approach, the truthlikeness of $h$ only depends on what $h$ says about the basic features of the world. These are independent facts which may or not obtain in the world (like "it's hot" and "it's dry") and are described by the basic propositions of $\mathcal{L}_{n}$. Accordingly, the key Popperian intuition above can be rephrased as follows: $h$ is close to the truth when $h$ provides much information about the basic features of the world and most of this information is true.

More formally, let $B(h)$ denote the set of "basic consequences" of $h$, i.e., the set of basic propositions (literals) entailed by $h$. Following Popper, we define the set $B_{T}(h)=B(h) \cap C n(t)$ of the true basic consequences of $h$, and the set $B_{F}(h)=B(h) \cap F$ its false basic consequences, to the effect that $B_{T}(h) \cup B_{F}(h)=B(h)$. Then, $h$ is at least as truthlike as $g$ iff:

$$
B_{T}(h) \supseteq B_{T}(g) \text { and } B_{F}(h) \subseteq B_{F}(g)
$$

Moreover, $h$ is closer to the truth than $g$ if at least one of the two above inclusion relations is strict. It is not difficult to check that this definition eschews the problem plaguing Popper's one, and satisfies all conditions $\mathrm{P} 1-\mathrm{P} 4$. However, it leaves most of the propositions of $\mathcal{L}_{n}$ incomparable in truthlikeness. Suppose for instance that $h$ is "rainy and windy", $g$ is "cold", and the weather is actually cold, rainy and windy. Then $B_{T}(h)=\{$ "rainy", "windy" $\}$, while $B_{T}(g)=\{$ "cold" $\}$. Given that $B_{T}(h)$ and $B_{T}(g)$ are set-theoretically incomparable, one cannot apply the above definition to assess the relative truthlikeness of $h$ and $g$.

To avoid this, one can introduce a simple measure of truthlikeness as follows. Let $\operatorname{cont}_{T}(h)=\left|B_{T}(h)\right| / n$ and $\operatorname{cont}_{F}(h)=\left|B_{F}(h)\right| / n$ denote, respectively, the normalized number of true and false basic propositions entailed by $h$. Then a "contrast measure" of truthlikeness is defined as follows (Cevolani, Crupi, and Festa 2011, p. 188): ${ }^{2}$

$$
v s_{b}(h)=\operatorname{cont}_{T}(h)-\operatorname{cont}_{F}(h)
$$

In words, the verisimilitude of $h$ is the difference between the amount of true and false information provided by $h$ about the basic features of the world. As one can show, measure $v s_{b}$ provides adequate assessments of the relative truthlikeness of the conjunctive propositions of $\mathcal{L}_{n}$, i.e., of conjunctions of basic propositions. For instance, in the example just considered above, "rainy and windy" $(h)$ correctly turns out to be more verisimilar than "cold"

\footnotetext{
${ }^{2}$ In that paper, we introduced measure measure $v s_{b}$ in the more general form $v s_{b}(h)=$ cont $_{T}(h)-\phi \operatorname{cont}_{F}(h)$, where $\phi>0$ expresses the relative weight of truth and falsity in assessing truthlikeness. Here, we shall only consider the special case $\phi=1$. The label "contrast measure" refers to Amos Tversky's "feature contrast" model of similarity between psychological stimuli.
} 
$(g)$, since $h$ entails two true basic propositions instead of only one, as $g$ does. However, measure $v s_{b}$ is inadequate for non-conjunctive propositions, as the following example shows.

Let Henry accept the disjunctive proposition $h$ that it will be cold or rainy, whereas Gloria believes $g$, that it will be hot or dry or still. If the truth $t$ is that the weather is actually cold, rainy and windy, then, intuitively, $h$ is closer to the truth than $g$. This is so not only because $h$ is true and $g$ is false; but also because $g$ is actually equivalent to $\neg t$, i.e., admits all possibilities except the truth itself. Still, it is easy to check that $B_{T}(h)=B_{T}(g)=\emptyset$ and $B_{F}(h)=B_{F}(g)=\emptyset$, and hence $h$ and $g$ are equally verisimilar, i.e., have the same degree of truthlikeness as a tautology. More generally, all "weak" propositions (like all disjunctive or conditional propositions) are assigned the same (null) degree of truthlikeness. The new truthlikeness measure presented in the following overcomes this limitation of our previous account while preserving the central ideas underlying it.

\subsection{From "categorical" to "partial" information}

The unsatisfactory result above is due to the naive definition of "information" we are employing. According to such definition, $h$ provides information about the world just in case $h$ logically entails some basic proposition of $\mathcal{L}_{n}$; otherwise, $h$ doesn't provide any information at all. This is, however, too restrictive: intuitively, it is clear that, for instance, "cold or rainy" does provide at least some information about the weather, although not so much information as that provided by "cold" (or "rainy") itself. In short, our "categorical" account of information is too crude to deliver a fine grained definition of verisimilitude.

A better account is however in sight. To this purpose, we mobilize some classical work concerning confirmation and partial entailment (cf. Carnap 1950 and Salmon 1969; see also Crupi and Tentori 2013 and Roche 2018, sec. 5). A proposition $h$ ("fully") entails another proposition $g$ when $g$ is true in all cases $h$ is, or, equivalently, when $R(h) \subseteq R(g)$, i.e., when the range of $h$ is included in that of $g$. Instead, $h$ "partially" entails $g$ when $g$ is true in most (but not necessarily all) the cases $h$ is. More precisely, $h$ partially entails $g$ when $h$ is "positively relevant" for $g$, i.e., when $h$ raises the proportion of cases in which $g$ is true (Salmon 1969, p. 63).

Let $m$ be the "logical" probability measure, i.e., the measure assigning the same degree of probability to all constituents of $\mathcal{L}_{n}$. Since there are $2^{n}$ constituents, each of them has probability $1 / 2^{n}$. Moreover, the probability of $h$ is simply the proportion of constituents entailing $h$ out of the total number of constituents: $m(h)=|R(h)| / 2^{n}$. It follows that if $b$ is a basic proposition, $m(b)=2^{n-1} / 2^{n}=1 / 2$. Assuming that $h$ is consistent (as we shall always assume in the following), the conditional logical probability of $g$ given $h$ is defined as usual, i.e., $m(g \mid h)=m(h \wedge g) / m(h)$. This means 
that $m(g \mid h)$ is the proportion of the cases (i.e., constituents) in which $g$ is true out of the total number of cases in which $h$ is true. Then, according to the "positive relevance" account of partial entailment, $h$ partially entails $g$ iff $m(g \mid h)>m(g)$. Note that, if $h$ (fully) entails $g$, then $h$ also partially entails $g$, since $m(g \mid h)$ takes its maximum value 1 , but not vice versa.

The notion of partial entailment provides us with a corresponding account of "partial" (vs. categorical) information. We shall say that $h$ provides partial information about $g$ just in case $h$ partially entails $g$. As far as basic propositions $b$ are concerned, this happens just in case $m(b \mid h)>1 / 2$. Accordingly, we call any $b$ such that $m(b \mid h)>1 / 2$ a "partial basic consequence" of $h$ and denote $P B(h)$ their class. Of course, any basic consequence of $h$-i.e., any basic proposition "fully" entailed by $h$-is also a partial basic consequence of $h$, but not vice versa. How much information does $h$ provide about its partial basic consequences? A natural measure would be the plain difference $m(b \mid h)-m(b)=m(b \mid h)-\frac{1}{2}$, which however varies between $-\frac{1}{2}$ and $\frac{1}{2}$. It is then sufficient to multiply this quantity by 2 in order to obtain the following normalized measure of the information provided by $h$ on $b$ :

$$
\inf (h, b)=2 \times\left(m(b \mid h)-\frac{1}{2}\right)
$$

Note that $\inf (h, b)$ varies between -1 (if $h \vDash \neg b$ ) and 1 (if $h \vDash b$, i.e., if $b$ is a plain basic consequence of $h$ ).

Now, using again Popper's definition as a benchmark, let us denote $P B_{T}(h)=P B(h) \cap T$ and $P B_{F}(h)=P B(h) \cap F$, respectively, the class of true and false partial basic consequences of $h$, i.e., the set of basic truths and of basic falsehoods partially entailed by $h$. Moreover, define $\inf _{T}(h)$ and $\inf _{F}(h)$, respectively, as follows:

$$
\inf _{T}(h)=\frac{1}{n} \times \sum_{b \in P B_{T}(h)} \inf (h, b) \quad \text { and } \quad \inf _{F}(h)=\frac{1}{n} \times \sum_{b \in P B_{F}(h)} \inf (h, b)
$$

i.e., as the normalized amount of information provided about the basic truths (resp. falsehoods) partially entailed by $h$.

Finally, the truthlikeness of $h$ is defined as the difference between the amounts of partial true and false information provided by $h$ about the basic features of the world:

$$
v s(h)=i n f_{T}(h)-i n f_{F}(h)
$$

The above measure is normalized between 1, the degree of truthlikeness of the truth $t$, and -1 , the truthlikeness of the "complete falsehood" $f$, i.e., the conjunction of the negations of the literals of $t$. The verisimilitude of a tautology, which is 0 , provides a sort of natural middle point, discriminating 
between those propositions $h$ for which the amount of partial true information exceeds the amount of partial false information, and hence $v s(h)>0$, and those for which the opposite is true, and hence $v s(h)<0 .^{3}$

Moreover, measure vs has a number of interesting features, to which we now turn. To illustrate them, let us come back to our weather example. Let $c$ ("cold"), $r$ ("rainy"), and $w$ ("windy") be the only primitive propositions of a simple meteorological language $\mathcal{L}_{3}$. Moreover, assume that the truth $t$ about the weather (in some location, at some time) is $c \wedge r \wedge w$.

First, note that measure $v s$ provides assessments of relative truthlikeness also for pairs of propositions that measure $v s_{b}$ cannot discriminate. Take for instance the two propositions $c \vee r$ ("cold or rainy") and $\equiv \neg c \vee \neg r \vee \neg w$ ("hot or dry or still") already considered above. Then one can check that $v s_{b}(c \vee r)=v s_{b}(\neg c \vee \neg r \vee \neg w)=0=v s_{b}(\top)$. However, $v s(c \vee r) \simeq 0.22>$ $-0.14 \simeq v s(\neg c \vee \neg r \vee \neg w)$, which is the intuitively right assessment.

Second, while "extending" measure $v s_{b}$ in the above sense, measure vs agrees with it as far as conjunctive propositions are concerned. This is because, if $h$ is a conjunction of literals, the amount of true and false partial information provided by $h$ reduces, respectively, to the plain (normalized) number of true and false basic propositions entailed by $h$; and hence $v s(h)$ reduces to $v s_{b}(h)$. To illustrate, consider two conjunctive propositions like $h \equiv \neg c \wedge r \wedge w$ and $g \equiv \neg c \wedge \neg r \wedge w$. Then it is clear that, on the one hand, $\inf _{T}(h)=\operatorname{cont}_{T}(h)=\frac{2}{3}$ and $\inf _{F}(h)=\operatorname{cont}_{F}(h)=\frac{1}{3}$; and that, on the other hand, $\inf _{T}(g)=\operatorname{cont}_{T}(g)=\frac{1}{3}$ and $\inf _{F}(g)=\operatorname{cont}_{F}(g)=\frac{2}{3}$. Accordingly, $v s(h)=v s_{b}(h)=\frac{1}{3}>-\frac{1}{3}=v s_{b}(g)=v s(g)$.

Third, measure vs meets most of the adequacy conditions discussed in the literature. The example just discussed shows that vs satisfies condition P1, i.e., is able to order falsehoods according to their relative verisimilitude, thus eschewing the issue trivializing Popper's definition. As for P2, it is easy to find cases of informative falsehoods which are more verisimilar than some uninformative truths: for instance, $v s(\neg c \wedge r \wedge w) \simeq 0.33>0.22 \simeq v s(c \vee r)$, even if the former proposition is false and the latter is true. Finally, stronger falsehoods may well be less verisimilar than weaker ones, thus respecting P4: for instance, it is easy to check that $v s(\neg c)=-\frac{1}{3}>-\frac{2}{3}=v s(\neg r \wedge \neg w)$.

Fourth, measure vs fails to meet the Popperian condition P3, according to which truthlikeness increases with content among truths. As an example of this violation, consider the following: $v s(c \vee \neg r)=0<0.05 \simeq v s(c \vee$ $\neg r \vee w$ ), even if the former (true) proposition entails the latter, and hence is more informative. This is a surprising result, since measure $v s$ was, so

\footnotetext{
${ }^{3}$ Note that here we could consider, as we did in the original account (Cevolani, Crupi, and Festa 2011, p. 188), a more general form of measure vs-i.e., $\inf _{T}(h)-\phi i n f_{F}(h)$ where parameter $\phi>0$ weighs the total amount of false information provided by $h$. In light of Theorem 1 from the next section, this would invite for a comparison between this weighted measure and Oddie's weighted average measure of truthlikeness (Oddie 2013, p. 1663). We leave this issue to another occasion.
} 
to speak, built on the top of measure $v s_{b}$, which satisfies P3 and encodes the key Popperian intuition that truthlikeness is a mixture of truth and content (cf. Cevolani, Crupi, and Festa 2011, pp. 187-8). Moreover, and interestingly, the above result has to be qualified. In fact, it is easy to check that vs meets P3 as far as conjunctive propositions are concerned: more precisely, it satisfies the following condition, which follows from P3 but it is strictly weaker than it:

P5 If $h$ and $g$ are true conjunctive propositions, and $h$ entails $g, h$ is closer to the truth than $g$.

As an example, $v s(c \wedge r)=\frac{2}{3}>\frac{1}{3}=v s(c)$. In short, adding true conjuncts to true conjunctions of basic propositions does increase truthlikeness. Still, in the general case the proposed account violates P3; as we shall see in the next section, there are deep and interesting reasons for why this is the case.

\section{Discussion and comparison with other accounts}

In the foregoing section, we introduced a new truthlikeness measure for propositional languages grounded in and extending our original basic feature approach to verisimilitude (Cevolani, Crupi, and Festa 2011; Cevolani, Festa, and Kuipers 2013). Such measure (vs) is an improvement on the original measure $\left(v s_{b}\right)$ insofar as it allows to compare any pair of propositions, instead of conjunctive propositions only, in terms of their relative truthlikeness. The new account retains the central Popperian intuition according to which the truthlikeness of $h$ increases the more true information, and the less false information, $h$ delivers about the truth. In particular, truthlikeness depends on the degree to which $h$ partially entails basic truths and basic falsehoods about the relevant domain.

With reference to the classification proposed by Schurz and Weingartner (2010), the proposed account seems clearly consequence-based and employs a conjunctive approach to theory representation, since propositions are identified with the sets of their partial basic consequences. For this reason, it is surprising that it violates condition P3: that logically stronger truths are more informative, and hence more truthlike, than logically weaker truths. This principle, which Oddie (2013) calls "the value of content for truths", was defended by Popper (1963) and is typically satisfied by content-based and consequence-based accounts, like that of Schurz and Weingartner (1987, 2010). As for the similarity based approaches, it is defended by Niiniluoto (1987) and rejected by Oddie $(1986,2013)$. As we shall see in a moment, the fact that our account here agrees with Oddie's one is no coincidence at all. 


\subsection{A surprising equivalence}

To understand the relations between our approach and other ones in the literature, let us first see how Oddie's account work. The starting point of the similarity approach is defining a measure $\lambda(w, t)$ of the likeness or closeness of an arbitrary constituent to the true constituent $t$ (Oddie 2013, sect. 5). In our propositional framework, one can simply define $\lambda(w, t)$ as the number of atomic propositions on which $w$ and $t$ agree, divided by $n$. In this way, one immediately obtains that $\lambda(w, t)=1$ iff $w$ is the truth itself, and that the complete falsehood $f$ is maximally distant from $t$, since $\lambda(t, f)=0$. The similarity approach assumes, in Schurz's terminology, a disjunctive view of propositions, representing $h$ as the set of possible worlds compatible with $h$ or, equivalently, as the disjunction of the constituents in its range $R(h)$. By considering the average closeness to the actual world of all worlds in $R(h)$, one obtains the so called Tichý-Oddie "average" measure of truthlikeness (Oddie 1986):

$$
v s_{a v}(h) \stackrel{d f}{=} \frac{\sum_{w \models h} \lambda(w, t)}{|R(h)|}
$$

where $|R(h)|$ is the number of constituents entailing $h$. This measure varies between $v s_{a v}(t)=1$ and $v s_{a v}(f)=0$; a tautology has an intermediate degree of truthlikeness $v s_{a v}(T)=\frac{1}{2}$. It is well known that the average account meets P1, P2, and P4, but violates P3, the value of content for truths: for instance, with reference again to our weather example, where $t$ is $c \wedge r \wedge w$, one can check that $v s_{a v}(c \vee \neg r)=0.5<0.57 \simeq v s_{a v}(c \vee \neg r \vee w)$, even if both propositions are true and the former is logically stronger than the latter. Interestingly, however, also the average measure, like $v s$, meets the value of content for truths as far as conjunctive propositions are concerned, i.e. P5, as it is easy to check.

These similarities between our proposed measure $v s$ and Oddie's measure $v s_{a v}$ are explained by the following result:

Theorem 1 Measures vs and vsav are ordinally equivalent.

This means that, for any pair of propositions $h$ and $g, v s(h)>v s(g)$ iff $v s_{a v}(h)>v s_{a v}(g)$. Indeed, we can even prove that $v s$ is a simple linear transformation of $v s_{a v}$, so that they are essentially the same measure: for any $h$

$$
v s(h)=2 \times v s_{a v}(h)-1
$$

The above result is surprising since the two accounts were originally based on radically different intuitions about truthlikeness. On the one hand, it confirms the idea, defended by Schurz and Weingartner (2010, pp. 422-3), that conjunctive, consequence-based accounts like the one proposed here are successful in accommodating both "content" and "likeness" intuitions - to the 
point, in fact, to recover the full account proposed by Oddie within the similarity approach. On the other hand, Theorem 1 shows that a consequencebased account can violate one central Popperian condition, i.e., P3, contrary to what Schurz and Weingartner (2010, p. 422) would like to assume.

In this connection, it is interesting to see how our account relates to the one proposed by Schurz and Weingartner $(1987,2010)$. Their idea is to retain Popper's consequence-based definition of truthlikeness while restricting the class of consequences which are relevant in assessing truthlikeness. Accordingly, each proposition $h$ is identified with the set of the "relevant elements" it entails. Then one can again follow Popper and say that $h$ is closer to the truth than $g$ iff, roughly, $h$ entails more true relevant elements and less false relevant elements than $g$ (Schurz and Weingartner 1987). Schurz and Weingartner (2010, sec. 5) improve on their previous, comparative account by defining a truthlikeness measure $v s_{S W}$ which appropriately weigh true and false elements and meets all requirements P1-P4. Even without considering the complete definitions (which are somehow technically intricate), an example will nicely illustrate the above point and give the flavor of Schurz and Weingartner's approach.

With reference to the usual weather example, let us consider the two propositions $h=c \vee \neg r$ ("cold or dry") and $g=c \vee \neg r \vee w$ ("cold or dry or windy"). Note that both $h$ and $g$ are true, and that $h$ logically entails $g$. According to Schurz and Weingartner (1987, p. 54), the only relevant elements entailed by $h$ and $g$ are, respectively, $h$ and $g$ themselves: these are their only weakest consequences besides trivial ones, like $T$ or irrelevant disjunctive weakenings of the original theories. Now, while both $h$ and $g$ only entail true relevant elements (i.e., themselves), $g$ is a proper disjunctive weakening on $h$ and hence should be assigned only a "fraction" of the truthlikeness degree of $h$ (Schurz and Weingartner 2010, p. 431). In fact, one can calculate that $v s_{S W}(h)=\frac{1}{6}>\frac{1}{9}=v s_{S W}(g){ }^{4}$ This assessment respects $\mathrm{P} 3$.

In our approach, things are different. Here, $h=c \vee \neg r$ provides exactly the same amount of information about a basic truth $(c)$ and a basic falsehood $(\neg r)$. Accordingly, these two amounts cancel each other out and the total degree of truthlikeness is $v s(h)=0$. As for $g=c \vee \neg r \vee w$, instead, the new true disjunct does provide some information about a basic truth $(w)$, if quite small, and this makes the overall truthlikeness increase: in fact, one can check that $v s(h)=0<0.05 \simeq v s(g)$. This violates $\mathrm{P} 3$ and shows that our measure is not ordinally equivalent to the one proposed by Schurz and Weingartner.

\footnotetext{
${ }^{4}$ The general formula for calculating the truthlikeness of disjunctions with both true and false literals is $\left.\frac{v}{k} \times \frac{(n-k+1) !}{n !}\right)$, where $k$ is the total number of disjuncts, $v$ is the number of true disjuncts, and $n$ is the number of atomic propositions of the language (Schurz and Weingartner 2010, p. 431).
} 


\subsection{Truthlikeness and logical strength}

As noted above, the truthlikeness measure proposed here violates the "value of content for truths" condition (P3), which is instead satisfied by both content-based (Miller, Kuipers), consequence-based (Popper, Schurz and Weingartner) and some similarity-based accounts (Niiniluoto). Such result can be evaluated in two ways. It will be read as a flaw of our account by those who think that P3 is indispensable as a principle governing truthlikeness assessments. If however the general validity of P3 is perceived as an open problem, our result counts as a new argument in favor of the view that weakening true theories need not make their truthlikeness decrease in the general case, but only as far as conjunctive theories are concerned. Without trying to assess here the debate on this point, we would like to draw the reader's attention on further principles that, like P3, P4, and P5, connect logical strength with truthlikeness by constraining the relative verisimilitude of pairs of propositions, one of which entails the other.

First, one may note that, among the fundamental principles $\mathrm{P} 1-\mathrm{P} 4$, P3 has a special status, being the strongest, i.e., the most restrictive one. This is clear if one compares P4 and P3: the former just requires that, among false propositions, truthlikeness doesn't not always covary with logical strength (leaving the possibility open that this happens for particular pairs of false propositions), while the latter demands that this must happen for true propositions. A weaker principle, comparable to $\mathrm{P} 4$, would be the following:

P6 If $h$ and $g$ are true, and $h$ entails $g, h$ may be closer to the truth than $g$.

Such principle is arguably an indispensable adequacy condition, and is indeed satisfied by all accounts on the market, including the one presented here.

Other ways of weakening P3 have to do with the logical form of the relevant propositions involved in the comparison. For instance, let us call "completely true" a disjunction of only true literals, like for instance $c, c \vee w$, $c \vee r \vee w$ in our weather example (note that, as for conjunctive propositions, we consider a single literal as a "degenerate" disjunction). Then one can consider the following condition:

P7 If $h$ and $g$ are completely true disjunctions, and $h$ entails $g, h$ is closer to the truth than $g$.

Since P3 entails P7 (but not vice versa), all accounts meeting P3 also satisfies P7. Interestingly, however, also our proposed measure $v s$, which fails to meet P3, satisfies P7 (as does the average measure, by Theorem 1). Thus, while truthlikeness does not covary in general with logical strength among true propositions, this is the case as far as completely true disjunctions are concerned. 
Let us now call a conjunctive proposition "completely false" just in case its conjuncts (literals) are all false (an example is $f$, the complete falsehood of the language). ${ }^{5}$ So for instance, in the weather example, $\neg c$ and $\neg c \wedge \neg r$ are completely false in this sense. Now the following requirement seems quite natural:

P8 If $h$ and $g$ are completely false conjunctive propositions, and $g$ entails $h$, $h$ is closer to the truth than $g$.

The above condition requires that truthlikeness increases with logical weakness among complete conjunctive falsehoods. Intuitively, this seems hardly disputable: in the above example, moving from $\neg c \wedge \neg r$ to $\neg c$ alone seems clearly an improvement in terms of truthlikeness. Indeed, this is what all accounts on the market deliver, with the exception of the content-based accounts, which violates $\mathrm{P} 4$ and hence also P8.

Finally, Ilkka Niiniluoto (personal communication) has attracted our attention on another condition which is rarely discussed in the literature (but see Schurz and Weingartner 1987, p. 65, for an exception). This corresponds to principle $\mathrm{P} 8$ above, but concerns false disjunctions of literals, i.e., disjunctions of false basic propositions only, like $\neg c$ and $\neg c \vee \neg w$ in our example. Now the principle corresponding to P 8 above becomes:

P9 If $h$ and $g$ are false disjunctions of basic propositions, and $g$ entails $h, h$ is closer to the truth than $g$.

For instance, P9 requires that moving from $\neg c$ to $\neg c \vee \neg w$ does improve truthlikeness. Is this principle defensible in general? Intuitions are less clear here than in the case of conjunctive propositions. Within the account proposed in this paper, a positive answer to the above question can be given along the following lines: both $\neg c$ and $\neg c \vee \neg w$ only provide false information about the world; however, the weaker theory provides a smaller amount of such false information, and hence it is more verisimilar. In fact, one can check that our account does meet condition P9. A similar diagnosis is given by Schurz and Weingartner (1987, 65, notation adapted): "if $g$ is stronger than $h$, both $h$ and $g$ are false and have no nontrivial true relevant consequences, then $g$ states a stronger relevant falsity than $h$ and has not more relevant truth content, hence $g$ should be considered more distant from the truth than $h . "$

Niiniluoto, however, disagrees (personal communication). From his similaritybased perspective, moving from $\neg c$ to $\neg c \vee \neg w$ does not improve our "best guess" about the truth (since the possible world $w$ closest to $t$ is at distance

\footnotetext{
${ }^{5}$ One may note that conjunction and disjunctions are "dual" to each other in the sense that a true conjunction is also "completely true" (but a false conjunction doesn't need to be completely false) whereas a false disjunction is also "completely false" (but a true disjunction doesn't need to be completely true).
} 
$\lambda(w, t)=\frac{1}{3}$ in both cases $)$ and it decreases the amount of information provided by the latter theory. Accordingly, $\neg c$ should be at least as close to the truth as $\neg c \vee \neg w$ and P9 should be rejected, which is what Niiniluoto's "minsum" measure of truthlikeness delivers. As convincing as one may find such argument, one should note that it follows from our Theorem 1 that also Oddie's similarity-based account meets P9. This suggests that similarity considerations alone are not enough to settle the question here.

In the end, we feel that further discussion is needed to properly assess the debate on the relations between truthlikeness and logical strength. Principles P3-9 surveyed here provide a reasonably complete and hopefully useful selection of conditions which might orientate such discussion in the future.

\section{Conclusions and further work}

In this paper, we proposed a new account of truthlikeness for propositional theories. In a nutshell, the truthlikeness of $h$ depends on the amount of true and false information provided by $h$ on the basic features of the world. The resulting measure improves on the one proposed in the original basic feature approach in being applicable to arbitrary, non-conjunctive propositions. Moreover it meets some important adequacy conditions discussed in the literature; in particular, it eschews both the trivialization result plaguing Popper's original definition and the child's play objection troubling contentbased accounts of truthlikeness.

The proposed account agrees with Popper, Schurz and Weingartner, and other proponents of consequence-based approaches on explicating truthlikeness in terms of true and false pieces of information entailed by different theories. Still, and surprisingly, it turns out to be equivalent to Oddie's wellknown similarity-based account. As a consequence, it violates the "value of content for truths" condition (P3), which is instead satisfied by both contentbased (Miller, Kuipers), consequence-based (Popper, Schurz and Weingartner) and some similarity-based accounts (Niiniluoto). On the other hand, it meets other, plausible conditions (surveyed in the previous section) concerning the interplay between truthlikeness and logical strength.

As noted by two anonymous reviewers, these features of our account raise interesting questions about how it should be accommodated within the classification of different approaches to truthlikeness introduced in section 2, and whether such classification is still tenable in the light of our results. Recall that, following Schurz, we started by considering two different ways of classifying existing accounts of truthlikeness: one based on a threefold classification among consequence, content, and likeness approaches, and one based on a twofold distinction between conjunctive and disjunctive views of theories. The latter distinction simply concerns how theories or propositions are represented, i.e., as conjunctions of content-parts or as disjunctions of 


\begin{tabular}{lcc} 
& conjunctive & disjunctive \\
\hline \multirow{2}{*}{ consequence } & Schurz and Weingartner & $?$ \\
content & $(1987,2010)$ & \\
& Cevolani (2016) & Kuipers $(1987,2000)$, \\
likeness & $?$ & Miller $(1978)$ \\
& & Niiniluoto (1987), \\
\hline
\end{tabular}

Table 1: Combining the three approaches to truthlikeness (rows) and the two approaches to theory representation (columns).

possibilities. The former classification is somehow vaguer, and has to do both with the method of constructing verisimilitude measures and with the basic principles such measures satisfy. In a nutshell, consequence approaches usually respect both principles $\mathrm{P} 3$ and P4; content approaches meet P3 and the negation of $\mathrm{P} 4$; and likeness approaches satisfy at least $\mathrm{P} 4$ and possibly (but not necessarily) P3.

Combined together, Schurz's distinctions would give raise to six possible combinations, corresponding to the six types of accounts displayed in table 1 along with some representative instances of them to be found in the literature. How should we classify our present account within the table? It is clear that we assumed a conjunctive representation of theories, so it must belong to the left column. Moreover, we defined truthlikeness in terms of true and false partial consequences, so our measure $v s$ is clearly an instance of consequence-based approaches, thus belonging to the top-left cell. ${ }^{6}$ On the other hand, in view of Theorem 1, measure vs seems also to belong to the class of conjunctive and likeness-based accounts in the bottom-left cell, which, as far as we know, was previously not instantiated in the literature. In fact, it violates principle P3, as only likeness-based accounts do (Oddie 2013), and it is equivalent to Oddie's measure, which is, by all standards, a canonical example of a likeness-based definition of truthlikeness.

The fact that the present account may appear both under the "consequence" and under the "likeness" heading in table 1 suggests that the three elements in the consequence/content/likeness classification are at best exhaustive but not exclusive. Moreover, the top-right cell in the table seems to remain empty. This would contain consequence-based accounts (defining truthlikeness as a matter of true and false consequences of theories) which employ a disjunctive representation of theories in terms of possible worlds (or constituents). To the best of our knowledge, no such account has been

\footnotetext{
${ }^{6}$ In addition, also the measures discussed in Cevolani, Crupi, and Festa (2011) and Cevolani, Festa, and Kuipers (2013), on which measure vs is based, clearly belong to the conjunctive, consequence-based category, even if they are only defined for so-called conjunctive theories (cf. section 3).
} 
proposed in the literature; also, it doesn't seem easy to imagine how it would work. This suggests, in turn, that the consequence/content/likeness and the conjunctive/disjunctive distinctions are not independent from each other (since it seems that consequence approaches can only be conjunctive as far as theory representation is concerned). How to evaluate this situation is still matter of debate. On the one hand, one may believe, as one of the reviewers does, that Schurz's distinctions, illustrated in table 1, are ill-founded and could be dispensed of. On the other hand, one can defend the resulting classification as an heuristically useful tool for better understanding the assumptions of different accounts and their relationships, as we did in this paper. Also in view of recent, ongoing work to make such classification mathematically more precise (cf. Oddie 2013), we hope that our present results will contribute to the future discussion on these issues.

Another, related debate has to do with the preferability of conjunctive over disjunctive accounts (or vice versa). Schurz and Weingartner (2010, pp. 423-4) claim that the former are more plausible than the latter, essentially because they are cognitively more natural and manageable. One reviewer asks how this can be true in general, in view of the fact (stated in Theorem 1) that our conjunctive account is equivalent to Oddie's disjunctive account. This is an interesting issue, which we cannot discuss here in full details. Two remarks, however, are in order.

First, the fact that two methods of measuring or calculating some quantity are equivalent in the sense of giving the same results (as measure $v s$ and Oddie's measure are) does not imply that they are "cognitively equivalent" in the sense of being equally transparent or their steps being equally accessible from a cognitive point of view. A familiar example comes from the so-called divisibility rules of arithmetic: for example, to check, say, whether a given (big) integer is divisible by 9 , one can either perform the required division or sum the digits of the number and verify whether the result is divisible by 9 . The two methods are equivalent, but the latter is much simpler and more easily applied. Second, the advantages of a conjunctive account of truthlikeness over a disjunctive one may become clear not so much when defining truthlikeness measures (what we did in this paper), but rather, when one employs these measures in order to assess the relative expected truthlikeness of two competing hypotheses on the basis of some given evidence. As far as our present account is concerned, we conjecture that it delivers an especially simple measure of expected truthlikeness, which can then be usefully applied to solve the so called epistemic problem of verisimilitude. The discussion of this conjecture, however, has to be left for another occasion.

Acknowledgments. We would like to thank Vincenzo Crupi, Theo Kuipers, Ilkka Niiniluoto, and Luca Tambolo for very useful discussions on the topics 
of this paper, and three anonymous reviewers for detailed critical comments on a previous draft. Gustavo Cevolani gratefully acknowledges financial support from the Italian Ministry of Education, Universities and Research (MIUR) through the FFABR 2017 grant.

\section{References}

Carnap, R. (1950). Logical foundations of probability. Chicago: University of Chicago Press. Second revised edition: 1962.

Cevolani, G. (2016). "Carnapian truthlikeness". In: Logic Journal of the IGPL 24.4, pp. 542-556.

- (2017). "Truthlikeness and the problem of measure sensitivity". In: EPSA15 Selected Papers. Ed. by M. Massimi, J.-W. Romeijn, and G. Schurz. Springer, pp. 257-271.

Cevolani, G., V. Crupi, and R. Festa (2011). "Verisimilitude and belief change for conjunctive theories". In: Erkenntnis 75.2, pp. 183-202.

Cevolani, G. and R. Festa (2018). "A partial consequence account of truthlikeness". In: Synthese. URL: 10.1007/s11229-018-01947-3.

Cevolani, G., R. Festa, and T. A. F. Kuipers (2013). "Verisimilitude and belief change for nomic conjunctive theories". In: Synthese 190.16, pp. 33073324 .

Crupi, V. and K. Tentori (2013). "Confirmation as Partial Entailment: A Representation Theorem in Inductive Logic". In: Journal of Applied Logic 11.4, pp. 364-372.

Kuipers, T. A. F. (1987). "A structuralist approach to truthlikeness". In: What is Closer-to-the-Truth? Ed. by T. A. F. Kuipers. Amsterdam: Rodopi, pp. 79-99.

- (2000). From Instrumentalism to Constructive Realism. Dordrecht: Kluwer Academic Publishers.

Miller, D. (1974). "Popper's qualitative theory of verisimilitude". In: The British Journal for the Philosophy of Science 25.2, pp. 166-177.

- (1978). "On distance from the truth as a true distance". In: Essays on Mathematical and Philosophical Logic. Ed. by J. Hintikka, I. Niiniluoto, and E. Saarinen. Dordrecht: Kluwer, pp. 415-435.

Niiniluoto, I. (1987). Truthlikeness. Dordrecht: Reidel.

- (1998). "Verisimilitude: the third period". In: The British Journal for the Philosophy of Science 49.1, pp. 1-29.

- (1999). Critical Scientific Realism. Oxford: Oxford University Press.

- (2003). "Content and likeness definitions of truthlikeness". In: Philosophy and Logic: in Search of the Polish Tradition. Essays in Honor of Jan Wolenski on the occasion of his 60th birthday. Ed. by J. Hintikka et al. Dordrecht: Kluwer Academic Publishers, pp. 27-35.

Oddie, G. (1986). Likeness to truth. Dordrecht: Reidel. 
Oddie, G. (2013). "The content, consequence and likeness approaches to verisimilitude: compatibility, trivialization, and underdetermination". In: Synthese 190.9, pp. 1647-1687.

- (2016). "Truthlikeness". In: The Stanford Encyclopedia of Philosophy. Ed. by E. N. Zalta. Winter 2016.

Popper, K. R. (1963). Conjectures and Refutations: the Growth of Scientific Knowledge. 3rd ed. London: Routledge and Kegan Paul.

Roche, W. (2018). "Is There a Place in Bayesian Confirmation Theory for the Reverse Matthew Effect?" In: Synthese 195, pp. 1631-1648.

Salmon, W. C. (1969). "Partial Entailment as a Basis for Inductive Logic". In: Essays in Honor of Carl G. Hempel. Ed. by N. Rescher. Reidel, pp. $47-82$.

Schurz, G. (2011). "Verisimilitude and Belief Revision. With a Focus on the Relevant Element Account". In: Erkenntnis 75.2, pp. 203-221.

Schurz, G. and P. Weingartner (1987). "Verisimilitude defined by relevant consequence-elements". In: What is Closer-to-the-Truth? Ed. by T. A. F. Kuipers. Amsterdam: Rodopi, pp. 47-77.

- (2010). "Zwart and Franssen's impossibility theorem holds for possibleworld-accounts but not for consequence-accounts to verisimilitude". In: Synthese 172, pp. 415-436.

Tichý, P. (1974). "On Popper's definitions of verisimilitude". In: The British Journal for the Philosophy of Science 25.2, pp. 155-160.

Zwart, S. D. (2001). Refined Verisimilitude. Dordrecht: Kluwer Academic Publishers.

Zwart, S. D. and M. Franssen (2007). "An impossibility theorem for verisimilitude". In: Synthese 158 (1), pp. 75-92.

\section{A Proofs}

In the following, we prove all the main claims in the paper.

- Measure vs meets P5. Let be $h$ and $g$ true, conjunctive propositions, i.e., conjunctions of true literals. It is then clear that $\inf _{F}(h)=$ $\inf _{F}(g)=0$, since both $h$ and $g$ partially entail no basic falsehoods. Moreover, $\inf _{T}(h)=\left|B_{T}(h)\right| / n$ and $\inf _{T}(g)=\left|B_{T}(g)\right| / n$, since both $h$ and $g$ "fully" entail each of their own conjuncts. Suppose now that $h$ entails $g$. This means that $B_{T}(h) \supset B_{T}(g)$, i.e., that $h$ adds to $g$ some true conjunct, and hence $\inf _{T}(h)>\inf f_{T}(g)$. It follows that $v s(h)=\inf _{T}(h)>i n f_{T}(g)=v s(g)$, i.e., that $\mathrm{P} 5$ is satisfied.

- Measure vsav meets P5. If $h$ is a conjunctive proposition, all constituents in the range of $h$ are "completions" of $h$, in the sense that they all agree with $h$ on each of its conjuncts. Now suppose that $h$ and $g$ are true, conjunctive propositions such that $h$ is logically stronger 
than $g$. This means that $h$ entails all (true) conjuncts of $g$ and also some other true conjunct. In turn, this means that, by the closeness measure $\lambda$, each constituent in the range of $h$ is closer to the truth than each constituent in that of $g$. It follows that the average truthlikeness is greater for $h$ than for $g$, i.e., that P5 is satisfied.

- Theorem 1: Measures vs and $v_{a v}$ are ordinally equivalent. In the following, we shall use $b$ to denote arbitrary an basic proposition (literal) of $\mathcal{L}_{n}, w$ to denote an arbitrary constituent, and $t$ for the true constituent. Moreover, in order to simplify notation, we shall write $r(h)$ for $|R(h)|$, i.e., for the number of constituents entailing $h$.

We start by noting that, for any $h$ and $b, m(\neg b \mid h)=1-m(b \mid h)$; from this and the definition of inf it follows that $\inf (h, \neg b)=-\inf (h, b)$. Accordingly, vs can be rewritten as follows:

$$
\begin{aligned}
v s(h) & =\inf _{T}(h)-\inf _{F}(h) \\
& =\frac{1}{n} \times \sum_{b \in P B_{T}(h)} \inf (h, b)-\frac{1}{n} \times \sum_{b \in P B_{F}(h)} \inf (h, b) \\
& =\frac{1}{n} \times \sum_{b \in P B(h): t \models b} \inf (h, b)-\frac{1}{n} \times \sum_{b \in P B(h): t \models \neg b} \inf (h, b) \\
& =\frac{1}{n} \times \sum_{b \in P B(h): t \models b} \inf (h, b)-\frac{1}{n} \times \sum_{b \notin P B(h): t \models b}-\inf (h, b) \\
& =\frac{1}{n} \times \sum_{b \in P B(h): t t \in b} \inf (h, b)+\frac{1}{n} \times \sum_{b \notin P B(h): t \models b} \inf (h, b) \\
& =\frac{1}{n} \times \sum_{b: t \models b} \inf (h, b)
\end{aligned}
$$

Moreover, by definition of inf:

$$
\begin{aligned}
v s(h) & =\frac{1}{n} \times \sum_{b: t \models b} \inf (h, b) \\
& =\frac{1}{n} \times \sum_{b: t \models b}\left(2\left(m(b \mid h)-\frac{1}{2}\right)\right) \\
& =\frac{2}{n} \times \sum_{b: t \neq b}\left(m(b \mid h)-\frac{1}{2}\right) \\
& =\frac{2}{n}\left(\sum_{b: t \models b} m(b \mid h)-\frac{n}{2}\right) \\
& =\frac{2}{n} \sum_{b: t=b} m(b \mid h)-1
\end{aligned}
$$

As for the average measure, note that, by definition of $v s_{a v} h$, of $\lambda(w, t)$, and of $B_{T}(w)$ :

$$
\begin{aligned}
v s_{a v}(h) & =\frac{1}{r(h)} \sum_{w: w \models h} \lambda(w, t) \\
& =\frac{1}{r(h)} \sum_{w: w \models h} \frac{\left|B_{T}(w)\right|}{n}
\end{aligned}
$$

Moreover, since for any constituent $w, r(b \wedge w$ ) is either 1 (if $w \vDash b$ ) or 0 (if $w \not \models b),\left|B_{T}(w)\right|$ can be written as $\sum_{b: t \models b} r(b \wedge w)$. Accordingly,

$$
\begin{aligned}
v s_{a v}(h) & =\frac{1}{r(h)} \sum_{w: w \models h} \frac{1}{n} \sum_{b: t \in b} r(b \wedge w) \\
& =\frac{1}{n} \sum_{w: w \models h} \sum_{b: t \models b} \frac{r(b \wedge w)}{r(h)}
\end{aligned}
$$

Now note that $m(b \mid h)=\frac{m(b \wedge h)}{m(h)}=\frac{r(b \wedge h)}{r(h)}$ by definition. In turn, $r(b \wedge h)$ can be written as $\sum_{w: w \models h} r(b \wedge w)$. It follows that $m(b \mid h)=$ $\sum_{w: w \models h} \frac{r(b \wedge w)}{r(h)}$ and hence that:

$$
v s_{a v}(h)=\frac{1}{n} \sum_{b: t \notin b} m(b \mid h)
$$


By the comparison of equations 1 and 2 above, it follows that:

$$
v s(h)=2 \times v s_{a v}(h)-1
$$

and hence that measures $v s$ and $v s_{a v}$ are ordinally equivalent, which completes the proof of Theorem 1.

- Measure vs meets P\%. We start by stating the following results, which will be useful in the following. First, if $h$ is a disjunction of $k$ literals, the amount of information provided by $h$ on each of its disjunct $b$ is:

$$
\begin{aligned}
\inf (h, b) & =2 \times\left(m(b \mid h)-\frac{1}{2}\right)=2 \times\left(\frac{m(b \wedge h)}{m(h)}-\frac{1}{2}\right) \\
& =2 \times\left(\frac{2^{n-1}}{2^{n}-2^{n-k}}-\frac{1}{2}\right)=\frac{1}{2^{k}-1}
\end{aligned}
$$

In fact, note that if $b$ is a disjunct of $h$, then $b \vDash h$ and $m(b \wedge h)=$ $m(b)=2^{n-1}$. Moreover, $h$ is false only in those worlds where all $k$ literals of $h$ are false; since there are $2^{n-k}$ such worlds, $m(h)=$ $2^{n}-2^{n-k}$.

Second, if $h$ is either completely true or false, its truthlikeness only depends on the total number $k$ of its disjunct and on the amount of information provided by $h$ on each of them (as just calculated above). In fact:

if $h$ is completely true, then $v s(h)=i n f_{T}(h)=\frac{1}{n} \times \frac{k}{2^{k}-1}$

if $h$ is false, then $v s(h)=-i n f_{F}(h)=-\frac{1}{n} \times \frac{k}{2^{k}-1}$

This is because if $h$ is completely true (resp. false), then it entails no basic falsehoods (resp. truths), and hence $\inf _{F}(h)\left(\right.$ resp. $\left.\inf _{T}(h)\right)$ is zero.

Finally, one should note that the factor $\frac{k}{2^{k}-1}$ appearing in the two expressions in 4 decreases for increasing $k$. To show this, we prove that increasing $k$ by 1 (i.e., weakening $h$ by the addition of one false disjunct) makes the above factor decrease:

$$
\begin{aligned}
\frac{k+1}{2^{k+1}-1} & <\frac{k}{2^{k}-1} & \text { iff } \\
(k+1)\left(2^{k}-1\right) & <k\left(2^{k+1}-1\right) & \text { iff } \\
2^{k}-1 & <k 2^{k} & \text { iff } \\
1-\frac{1}{2^{k}} & <k &
\end{aligned}
$$

Recalling that $k$ is a positive integer $(k \geq 1)$, the above inequality is always satisfied, which proves that $\frac{k}{2^{k}-1}$ decreases for increasing $k$.

Now, coming back to P7, let be $h$ and $g$ two completely true disjunctions of literals such that $h \vDash g$. This means that $g$ weakens $h$ by adding to it some true basic disjunct, in the sense that $g$ has the form 
$h \vee b$ for at least one $b$ different from the disjuncts of $h$. Since the number $k$ of disjuncts increases by moving from $h$ to $g$, truthlikeness decreases by equations 4 and 5 above, and then $h$ is more verisimilar than $g$, which proves that vs meets $\mathrm{P} 7$.

- Measure vs meets P8. Let be $h$ and $g$ completely false conjunctive propositions, i.e., conjunctions of false literals. It is then clear that $\inf _{T}(h)=\inf _{T}(g)=0$, since both $h$ and $g$ partially entail no basic truths. Moreover, inf ${ }_{F}(h)=\left|B_{F}(h)\right| / n$ and $\inf _{F}(g)=\left|B_{F}(g)\right| / n$, since both $h$ and $g$ "fully" entail each of their own conjuncts. Suppose now that $h$ entails $g$. This means that $B_{F}(h) \supset B_{F}(g)$, i.e., that $h$ adds to $g$ some false conjunct, and hence that $\inf _{F}(h)>\inf f_{F}(g)$. It follows in turn that $v s(h)=-i n f_{F}(h)<-i n f_{F}(g)=v s(g)$, i.e., that $\mathrm{P} 8$ is satisfied.

- Measure vs meets P9. Let be $h$ and $g$ two false disjunctions of literals such that $h \vDash g$. This means that $g$ has a greater number of disjuncts than $h$ (compare the proof that vs meets $\mathrm{P} 7$ above). From equations 4 and 5 above, it then follows that $\inf _{F}(g)<i n f_{F}(h)$ and hence that $v s(g)=-i n f_{F}(g)>-i n f_{F}(h)=v s(h)$, i.e., that $g$ is more verisimilar than $h$, which proves that vs meets $\mathrm{P} 9$. 